\title{
Multitasking in Gonadotropin-Releasing Hormone Neuron Dendrites
}

\author{
Karl J. Iremonger Allan E. Herbison \\ Centre for Neuroendocrinology, Department of Physiology, University of Otago School of Medical Sciences, \\ Dunedin, New Zealand
}

\section{Key Words}

Gonadotropin-releasing hormone · Median eminence · Neuron dendrite · Dendron

\begin{abstract}
Gonadotropin-releasing hormone ( $\mathrm{GnRH}$ ) neurons integrate synaptic information in their dendrites in order to precisely control GnRH secretion and hence fertility. Recent discoveries concerning the structure and function of GnRH neuron dendrites have shed new light on the control of GnRH neuron excitability and $\mathrm{GnRH}$ secretion. This work suggests that GnRH neurons have a unique projection to the median eminence that possesses both dendritic and axonal properties. We propose that this 'dendron' projection allows GnRH neurons to multitask and integrate information in ways that would not be possible in a classically envisioned axon projection.

(c) 2014 S. Karger AG, Basel
\end{abstract}

\section{Introduction}

Gonadotropin-releasing hormone (GnRH) neurons regulate puberty and fertility in all mammalian species. These neurons project to the median eminence where $\mathrm{GnRH}$ is secreted to control pituitary function. The excitability of GnRH neurons and hence the secretion of GnRH peptide are controlled by a distributed and complex neural network. While increasingly more is known about the con- nectivity and function of the upstream neurons that project to GnRH neurons, this review will focus specifically on how GnRH neurons are adapted to integrate and process afferent synaptic inputs. More specifically, we will focus on the dendritic specializations that make this possible. This knowledge has been synthesized through a variety of methods including morphological, electrophysiological, functional imaging and computational studies. Together, this work suggests that GnRH neurons have morphological features that allow them to perform multiple operations in a unique median eminence projecting fibre.

\section{GnRH Neuron Projections: Long and Spiny}

It should not come as a surprise that GnRH neurons appear and function differently to other central nervous system neurons since the embryonic origins of these neurons are quite unique. Specifically, GnRH neurons are born in the olfactory placode and then migrate into the brain during embryonic development [1]. The cell bodies of GnRH neurons in the adult are scattered along this migration path from the olfactory bulb through to the median eminence. However, the majority of GnRH neurons reside in the medial septum, rostral pre-optic area (rPOA) and anterior hypothalamic area (AHA) [2]. While GnRH neurons from all of these areas project to the median eminence to release $\mathrm{GnRH}$ peptide into the portal circulation, the complete morphology of these neurons has only recently been revealed [3].

\section{KARGER 125}

(c) 2014 S. Karger AG, Base

$0028-3835 / 14 / 1022-0001 \$ 39.50 / 0$ 
GnRH neurons typically have two processes, each originating from opposite poles of the soma and each possessing dendritic spines [4-9]. Morphological reconstructions of GnRH neurons in acute brain slices have revealed that these dendrites project for large distances in the brain [10-12]. Further studies have revealed that this simple dendritic morphology is acquired over the course of puberty, since the morphology of GnRH neurons reconstructed from rodents before puberty is significantly more complex, with many more dendritic branches [13, 14].

Similar to many other central neurons, GnRH neuron dendrites also possess dendritic spines [4-9]. The density of these spines is highest on the proximal $50 \mu \mathrm{m}$ of dendrite and steadily declines with increasing distance from the soma [10]. Notably however, dendritic spines are still present hundreds of micrometres from the soma [10] suggesting the synaptic inputs are received even at very distant sites.

The individual dendrites of GnRH neurons also come into close contact with one another. This arrangement, termed bundling, involves the intertwining of dendrites from different GnRH neurons over short distances. These bundled regions are often connected via zonula adherens and also have shared synapses [12]. Evidence suggests that this bundling is extensive amongst $\mathrm{GnRH}$ neuron dendrites and is one potential mechanism by which this scattered population could be coordinated. Interestingly, adult GnRH neurons from the mouse do not appear to possess gap junctions [15]. Therefore coordination of activity must employ other mechanisms rather than direct electrical coupling via dendritic gap junction channels. Possible, but yet untested mechanisms include electrical coupling via ephaptic interactions [16] or coordination via dendritically released neurotransmitters $[17,18]$. One alternative possibility is that bundling is simply a byproduct of dendrite path finding during embryonic development and in fact plays no role in electrical coordination at all. If this was the case, coordinated activity amongst GnRH neurons may simply be driven by coordinated excitatory inputs from upstream neural populations.

\section{Do the Dendrites Project All the Way to the Median Eminence?}

Morphological reconstructions had demonstrated that GnRH neurons have long, thin dendrites but failed to identify the axonal projection to the median eminence.
This led to speculation that the long dendrites of these neurons were in fact the primary projection to the median eminence.

Reconstructions of the entire GnRH neuron were previously hindered by the fact that the projections are severed during the preparation of thin acute brain slices. To circumvent this problem, a thick ventral-horizontal brain slice preparation was developed that maintains the ventral most population of GnRH neurons with their entire projections to the median eminence intact [19].

Full reconstructions of GnRH neurons in these thick brain slices revealed that the previously termed dendrites of these neurons indeed project all the way to the median eminence [3]. While these projections neither label for classical axonal or dendritic markers, they do however possess dendritic spines and presynaptic appositions along their entire length [3]. Upon reaching the border of the median eminence, these processes branch extensively and take on an axonal appearance. These axon-like processes eventually form neurosecretory terminals apposed to blood vessels in the external zone of the median eminence [3]. This unique projection structure has been named the 'dendron' because it appears to function simultaneously as a dendrite and axon [3]. Because GnRH neurons typically have two dendrites, it was important to determine if each dendrite projected to the same or different locations. Remarkably, in AHA GnRH neurons where both dendrons have been able to be fully reconstructed, it has been found that both project into the median eminence [3]. It is currently unknown whether the two dendrons originating from opposite sides of the soma function identically or differently.

To date, only the projections of GnRH neurons within the AHA have been fully reconstructed, the reason being that an acute brain slice containing the rPOA with intact projections to the median eminence is not currently feasible. Alternative approaches such as cell filling of rPOA GnRH neurons in vivo will instead have to be employed to study the projection of these neurons. However, based on the morphology and direction of travel of the proximal dendrite of rPOA GnRH neurons, it is expected that these neurons will also possess the dendron projection to the median eminence. It is important to note that there exists a population of $\mathrm{GnRH}$ neurons that also send projections to other central brain regions such as the periaqueductal grey, amygdala and cortex [8,20-24]. It is likely that this small population of centrally projecting $\mathrm{GnRH}$ neurons possesses an axonal projection in addition to their median eminence-targeting dendron projection. 


\section{Multitasking in the Dendron}

Morphological reconstructions suggest that GnRH neurons send a projection to the median eminence that has a hybrid dendritic-axonal structure. If this is indeed the case, this projection should possess functional properties of both axons and dendrites. Specifically, it should both be able to initiate and actively propagate action potentials in addition to receiving and integrating synaptic inputs.

The ability of GnRH neuron dendrites to initiate and propagate spikes was first demonstrated by the Suter laboratory [25]. By performing whole-cell recordings at the soma and on-cell recordings along the dendrite, Roberts et al. [25] demonstrated in 2008 that the dendrites of GnRH neurons conduct spikes and are the site of spike initiation in the majority of recorded neurons. Subsequent work from our laboratory has confirmed this and further determined that these sodium spikes are actively conducted throughout all parts of the dendritic projection. In addition, we have identified a region $50-150 \mu \mathrm{m}$ down one dendrite as being the spike initiation site [26]. This region of the dendrite also labels for the spike initiation site marker ankyrin G [3] and possesses a high density of functional voltage-gated sodium channels [26]. Interestingly, while the entire dendritic projection can actively conduct spikes, there appears to be only one site of spike initiation. The soma and dendrites of GnRH neurons also possess many other voltage-gated ion channels which are known to be essential for the regulation of excitability and the generation of burst firing. For an indepth review of these ion channels, please see Moenter [27] and Norberg et al. [28].

It is clear from previous work that the proximal $\mathrm{GnRH}$ neuron dendrite receives a significant degree of synaptic innervation $[29,30]$. Remarkably, synaptic inputs onto the dendron exist along the entire projection to the median eminence. Specifically, we have found that the dendron projection possesses dendritic spines along its length, as well as juxtaposed vesicle-associated membrane protein 2 (VAMP2) immunostained puncta [3]. VAMP2 is a presynaptic vesicle protein and therefore indicates the presence of presynaptic terminals. To address whether activation of postsynaptic receptors on the dendron regulates excitability and spiking, we and others have used three different approaches: local neurotransmitter puffs, neurotransmitter uncaging and channelrhodopsin stimulation.

Neurotransmitter puffs onto the dendron evoke reliable membrane potential changes recorded at the cell body [26]. As expected for passive electrotonic decay of subthreshold potentials, the amplitude of these responses becomes smaller and smaller at greater distances from the recording electrode at the soma. The effects on spiking excitability are similar. Specifically, activation of glutamate receptors close to the spike initiation site readily evokes a burst of spiking. However, glutamate puffs at distances greater than $400-500 \mu \mathrm{m}$ from the soma are ineffective at inducing spiking [3]. It is important to note that glutamate-evoked sodium spikes are initiated at the same spike initiation site regardless of where along the dendron the glutamate receptors are activated. This location of spike initiation is the same as for spontaneously evoked spiking [26].

Laser uncaging of neurotransmitters is another way to study the impact of synaptic signals on dendrite excitability. Dendritic uncaging of either $\gamma$-aminobutyric acid (GABA) or glutamate can evoke depolarizations in $\mathrm{GnRH}$ neuron dendrites [31]. Interestingly, uncaging of GABA results in the activation of dendritic L-type voltage-gated calcium channels, which enhances the subsequent depolarization and spiking [31].

As noted above, activation of glutamate receptors at more distal regions of the dendron is ineffective at evoking spiking. We therefore investigated the possibility that subthreshold synaptic inputs may modify the shape of action potentials as they propagate along the dendron. Previous studies in hippocampal neurons have demonstrated that subthreshold depolarizations in the axon can broaden action potentials leading to changes in neurotransmitter release [32-35]. To investigate whether this occurs in GnRH dendrons, we focally uncaged glutamate while recording dendritic spikes with an on-cell recording electrode. Consistent with previous studies, we found that uncaging glutamate increased spike width without changing spike amplitude [3]. The implications of this phenomenon for functioning in the GnRH neuron will be discussed in a later section.

While both puffing and uncaging neurotransmitters provide evidence for functional postsynaptic receptors, they do not confirm whether endogenous neurotransmitter release occurs. To confirm that synapses onto the $\mathrm{GnRH}$ dendron are in fact functional, we have used channelrhodopsin 2 stimulation of GABA neurons [36]. When blue light is directed at channelrhodopsin-2expressing GABA neurons, these neurons are depolarized, fire action potentials and subsequently release neurotransmitter. Using this technique, we have also confirmed that $\mathrm{GnRH}$ dendrons receive functional GABAergic synaptic innervation [3]. 


\section{Functional Role of a Mixed Dendritic-Axonal (Dendron) Projection}

The total length of the GnRH neuron projection to the median eminence can be greater than $3 \mathrm{~mm}$ depending on whether the neuron is located in the AHA, rPOA or medial septum. The unique morphology and function of the GnRH projection to the median eminence suggest that these neurons integrate and process synaptic information differently to other neurons. Very broadly, we propose that the dendron has three functional compartments, with each performing specific computational roles: (1) proximal dendron, (2) central dendron and (3) distal dendron (fig. 1). Together, these compartments allow for at least three distinct modes of operation to control GnRH secretion from the median eminence.

\section{Proximal Dendron (Up to approx. $500 \mu \mathrm{m}$ from Cell Body)}

This is the best-studied region of the GnRH neuron. As noted earlier, this region contains the spike initiation site [26] and also has the highest density of synaptic innervation [10]. The synaptic inputs that impinge close to the spike initiation site will be highly effective at controlling action potential firing. Synaptic inputs at greater distances away from the spike initiation site will become progressively less effective at controlling spiking simply due to increased electronic decay [3]. While the length constant of GnRH neuron dendrites has not been directly measured, it may be similar to the axonal length constant of other neurons $(420-550 \mu \mathrm{m}[34,35,37])$. This predicts that the amplitude of synaptic inputs at approximately $500 \mu \mathrm{m}$ will be $37 \%$ of their original amplitude by the time they propagate back to the soma. Synaptic inputs that arrive at distances $>500 \mu \mathrm{m}$ (in the central and distal dendron) are predicated to be attenuated so much that they have minimal direct effects on somatic excitability.

Burst generation in GnRH neurons is thought to require ionic conductances that are highly expressed in the soma $[38,39]$. Therefore, there are implications for burst generation of having a spike initiation site in the proximal dendron. Modelling has demonstrated that the degree of electrical coupling between the site of spike initiation and the soma indeed plays an important role in regulating bursting behaviour [40]. Factors that increase electrical coupling (e.g. reduced distance between soma and spike initiation site) increase interburst interval and reduce the number of spikes per burst [40].

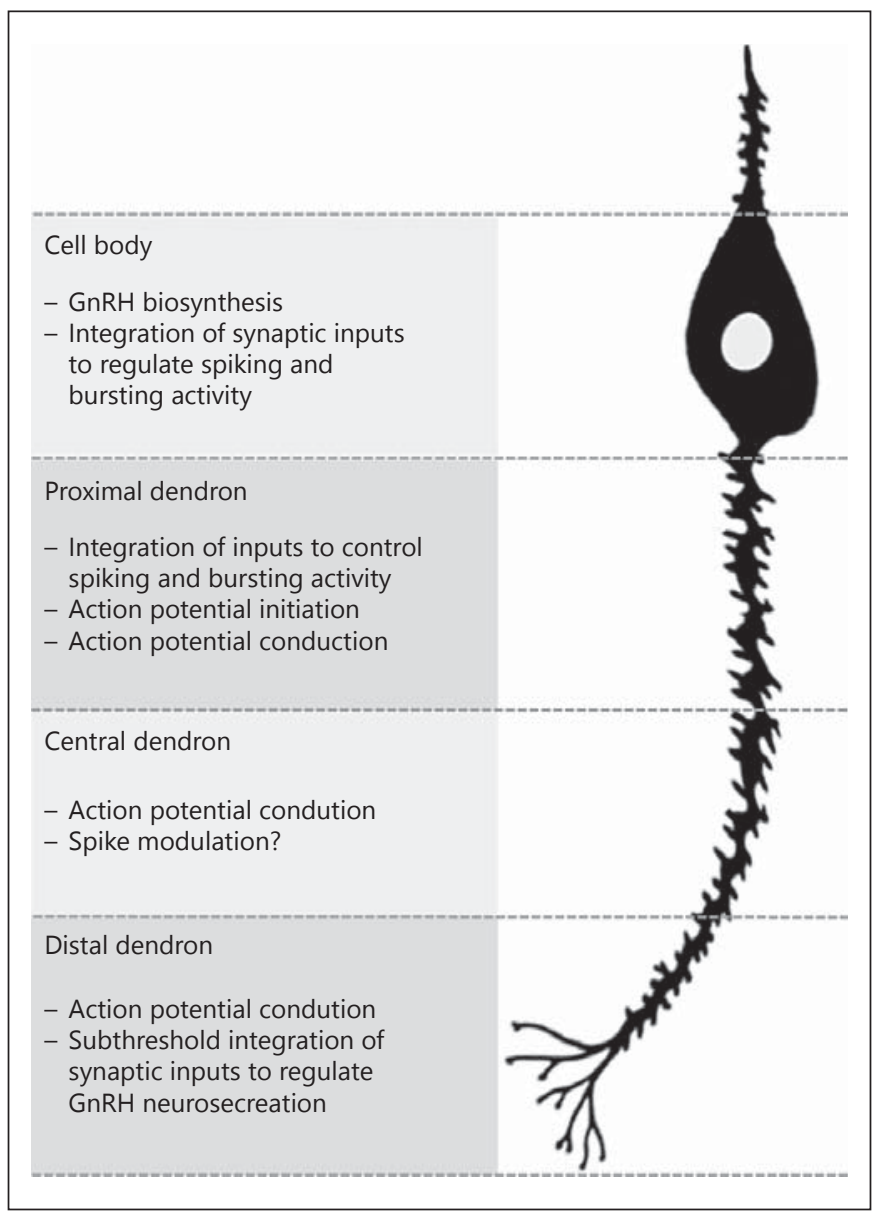

Fig. 1. Functional compartments of the GnRH neuron projection to the median eminence. The GnRH neuron can be functionally subdivided into four compartments: (1) the cell body controls gene transcription/translation and $\mathrm{GnRH}$ biosynthesis; it also receives synaptic inputs and has many of the ion channels necessary to generate electrical bursting activity; (2) the proximal dendron has the action potential initiation site as well as a high density of synaptic innervation; this region of the dendron is therefore critical for the generation and regulation of spiking activity; (3) the central dendron also actively conducts spikes and receives synaptic inputs; while synaptic inputs can modulate the shape of locally propagating spikes, the precise role of this region in regulating $\mathrm{GnRH}$ secretion is unclear; (4) the distal dendron conducts action potentials into the GnRH nerve terminals in the median eminence; this region also receives synaptic inputs, which are thought to regulate the membrane potential and calcium levels in the nerve terminals via subthreshold mechanisms.

Modelling has also demonstrated that the proximal dendron plays an important role in regulating the magnitude of afterdepolarization potentials [41] which are also thought to be important in regulating bursting activity [42]. 
Central Dendron (Region between Proximal and

Distal Dendron)

The functional importance of synaptic innervation in this central region of the GnRH neuron projection is currently unclear. As mentioned above, it has been shown that synaptic depolarizations are capable of regulating the shape of propagating spikes in this region. It is important to note however that this effect on the shape of the spike will only be localized to the region of the dendron where the synaptic input occurs. At regions further down the projection, where the depolarization will be very small or negligible, the impact on spike shape will cease to exist [33]. Therefore, current evidence suggests that synaptic inputs onto this central region will not impact excitability either at the spike initiation site or the nerve terminals. Thus, the functional significance of inputs in this region remains to be determined.

\section{Distal Dendron (Distal approx. $500 \mu \mathrm{m}$ of Projection)}

Perhaps the most significant implication for $\mathrm{GnRH}$ neuron processing exists in the distal dendron. Synaptic inputs that are received in this region will be too far away from the spike initiation site to control action potential firing. In addition, there is no current evidence to suggest that spikes can be locally generated in this distal region of the $\mathrm{GnRH}$ dendron either. Instead, we propose that this region of the GnRH neuron is specialized to integrate subthreshold synaptic signals, which can then be relayed to the nearby nerve terminals. In fact, there is already considerable evidence for direct control of $\mathrm{GnRH}$ release via integration of signals in $\mathrm{GnRH}$ processes close to the median eminence [43]. This includes studies that have demonstrated spontaneous pulsatile secretion of $\mathrm{GnRH}$ from isolated mediobasal/median eminence explants [44-46]. In addition, other studies have demonstrated that neurotransmitters and neuropeptides implicated in the central regulation of fertility can induce GnRH secretion in the absence of GnRH cell bodies [46, 47]. Topically, kisspeptin or neurokinin B can induce GnRH secretion when applied to isolated median eminence slices in vitro [48-51]. The effects of kisspeptin appear to be calcium dependent [50] but action potential independent [48]. Together, these data support the notion that activation of receptors on the distal dendron induces subthreshold depolarizations and calcium influx that can regulate $\mathrm{GnRH}$ secretion in a spike-independent manner. This mechanism of regulation could operate independently or synergistically with control mechanisms present in the proximal dendron/soma. For example, synaptic inputs onto the distal dendron could induce depolarizations/calcium influx to drive GnRH release. However, if depolarization of the distal dendron occurred coincidently with a burst of spiking initiated at the soma/proximal dendron, then GnRH release could be potentiated. Therefore, at least three distinct modes of operation are envisaged: (1) somatic spiking-induced GnRH release, (2) GnRH release driven solely by subthreshold inputs in the distal dendron and (3) synergistic release driven by coincident distal subthreshold and spiking signals.

A big question that now arises is whether the pulse and surge modes of GnRH secretion in vivo are due to the different modes of operation of the dendron. $\mathrm{c}$-fos data suggests that the cell body of $\mathrm{GnRH}$ neurons experiences a high level of spiking activity during the GnRH surge [5254], but could pulsatile GnRH release be driven by spikeindependent mechanisms in the distal dendron?

\section{Is this Form of Information Processing Unique to the GnRH Neuron?}

GnRH neurons clearly function differently from most other neurons in the mammalian brain. However, mounting evidence suggests even 'typical' neurons can perform dendrite-like computations locally within long-range axonal projections [55].

For example, synaptically released glutamate has been shown to regulate hippocampal axonal excitability via axonal kainate [56] and AMPA receptors [32]. In the cerebellum, a similar situation exists for locally released GABA onto parallel fibre axons [57]. Synaptic events that occur in dendrites can also passively propagate for very long distances down axons $[34,37]$. These subthreshold depolarizations can propagate to the nerve terminals and subsequently regulate nerve terminal excitability and neurotransmitter release. While this is a recently described form of information processing in central neurons, it has been known for a long time that this mechanism of signalling is commonly used in the retina [58] as well as in various invertebrate neurons $[59,60]$. Clearly, specialised forms of information processing are evident in diverse classes of neurons.

\section{Conclusion}

The dendron projection greatly expands the dynamic control of $\mathrm{GnRH}$ neuron excitability and hence $\mathrm{GnRH}$ secretion from the median eminence. Because of its ex- 
tensive length, we speculate that synaptic inputs received in different regions of the dendron regulate GnRH secretion via quite different mechanisms. Synaptic inputs received in the proximal dendron, close to the soma, will efficiently regulate spiking activity. In comparison, synaptic inputs onto the distal dendron, close to the nerve terminals, could regulate $\mathrm{GnRH}$ release via subthreshold changes in membrane potential and calcium levels. The next challenge is to identify the phenotype of the synaptic inputs along this unique projection and to determine how inputs at different locations control the profile of $\mathrm{GnRH}$ secretion in the whole animal.

\section{References}

1 Wray S: From nose to brain: development of gonadotrophin-releasing hormone-1 neurones. J Neuroendocrinol 2010;22:743-753.

2 Herbison AE: Physiology of the adult GnRH neuronal network; in Plant TM, Zeleznik AJ (eds): Knobil and Neill's Physiology of Reproduction, ed 4. San Diego, Academic Press, 2014

3 Herde MK, et al: GnRH neurons elaborate a long-range projection with shared axonal and dendritic functions. J Neurosci 2013;33 12689-12697.

4 Jennes L, Stumpf WE, Sheedy ME: Ultrastructural characterization of gonadotropinreleasing hormone $(\mathrm{GnRH})$-producing neurons. J Comp Neurol 1985;232:534-547.

5 Lehman MN, et al: Immunocytochemical localization of luteinizing hormone-releasing hormone (LHRH) pathways in the sheep brain during anestrus and the mid-luteal phase of the estrous cycle. J Comp Neurol 1986;244:19-35.

-6 Liposits Z, Setalo G, Flerko B: Application of the silver-gold intensified $3,3^{\prime}$-diaminobenzidine chromogen to the light and electron microscopic detection of the luteinizing hormone-releasing hormone system of the rat brain. Neuroscience 1984;13:513-525.

7 Witkin JW: Effects of ovariectomy on GnRH neuronal morphology in rhesus monkey ( $\mathrm{Ma}$ caca mulatta). J Neuroendocrinol 1996;8: 601-604.

8 Witkin JW, Paden CM, Silverman AJ: The luteinizing hormone-releasing hormone (LHRH) systems in the rat brain. Neuroendocrinology 1982;35:429-438.

-9 Wray S, Hoffman G: Postnatal morphological changes in rat LHRH neurons correlated with sexual maturation. Neuroendocrinology 1986;43:93-97.

10 Campbell RE, Han SK, Herbison AE: Biocytin filling of adult gonadotropin-releasing hormone neurons in situ reveals extensive, spiny, dendritic processes. Endocrinology 2005; 146 : 1163-1169.

11 Herde MK, et al: Gonadotropin-releasing hormone neurons extend complex highly branched dendritic trees outside the bloodbrain barrier. Endocrinology 2011;152:38323841.

12 Campbell RE, et al: Dendro-dendritic bundling and shared synapses between gonadotropin-releasing hormone neurons. Proc Nat Acad Sci U S A 2009;106:10835-10840.
13 Cottrell EC, et al: Postnatal remodeling of dendritic structure and spine density in gonadotropin-releasing hormone neurons. Endocrinology 2006;147:3652-3661.

14 Ybarra N, et al: Spatially selective, testosterone-independent remodeling of dendrites in gonadotropin-releasing hormone $(\mathrm{GnRH})$ neurons prepubertally in male rats. Endocrinology 2011;152:2011-2019.

15 Campbell RE, et al: Gap junctions between neuronal inputs but not gonadotropin-releasing hormone neurons control estrous cycles in the mouse. Endocrinology 2011;152:22902301.

16 Holt GR, Koch C: Electrical interactions via the extracellular potential near cell bodies. J Comput Neurosci 1999;6:169-184.

17 Chu Z, Moenter SM: Endogenous activation of metabotropic glutamate receptors modulates GABAergic transmission to gonadotropin-releasing hormone neurons and alters their firing rate: a possible local feedback circuit. J Neurosci 2005;25:5740-5749.

18 Iremonger KJ, Wamsteeker Cusulin JI, Bains JS: Changing the tune: plasticity and adaptation of retrograde signals. Trends Neurosci 2013;36:471-479.

19 Constantin S, et al: GnRH neuron firing and response to GABA in vitro depend on acute brain slice thickness and orientation. Endocrinology 2012;153:3758-3769.

20 Badr M, Pelletier G: Characterization and autoradiographic localization of LHRH receptors in the rat brain. Synapse 1987;1:567-571.

21 Boehm U, Zou Z, Buck LB: Feedback loops link odor and pheromone signaling with reproduction. Cell 2005;123:683-695.

22 Buma P: Characterization of luteinizing hormone-releasing hormone fibres in the mesencephalic central grey substance of the rat. Neuroendocrinology 1989;49:623-630.

23 Liposits Z, Setalo G: Descending luteinizing hormone-releasing hormone (LH-RH) nerve fibers to the midbrain of the rat. Neurosci Lett 1980;20:1-4.

24 Sakuma Y, Pfaff DW: Mesencephalic mechanisms for integration of female reproductive behavior in the rat. Am J Physiol 1979;237: R285-R290.

25 Roberts CB, et al: Dendritic action potential initiation in hypothalamic gonadotropinreleasing hormone neurons. Endocrinology 2008;149:3355-3360.
26 Iremonger KJ, Herbison AE: Initiation and propagation of action potentials in gonadotropin-releasing hormone neuron dendrites. J Neurosci 2012;32:151-158.

27 Moenter SM: Identified GnRH neuron electrophysiology: a decade of study. Brain Res 2010;1364:10-24.

28 Norberg R, Campbell R, Suter KJ: Ion channels and information processing in $\mathrm{GnRH}$ neuron dendrites. Channels (Austin) 2013;7: 135-145.

29 Iremonger KJ, et al: Glutamate regulation of GnRH neuron excitability. Brain Res 2010; 1364:35-43.

30 Clarkson J, Herbison AE: Development of GABA and glutamate signaling at the GnRH neuron in relation to puberty. Mol Cell Endocrinol 2006;254-255:32-38.

31 Hemond PJ, et al: Simulated GABA synaptic input and L-type calcium channels form functional microdomains in hypothalamic gonadotropin-releasing hormone neurons. J Neurosci 2012;32:8756-8766.

32 Sasaki T, Matsuki N, Ikegaya Y: Action-potential modulation during axonal conduction. Science 2011;331:599-601.

33 Sasaki T, Matsuki N, Ikegaya Y: Effects of axonal topology on the somatic modulation of synaptic outputs. J Neurosci 2012;32:28682876.

34 Shu Y, et al: Modulation of intracortical synaptic potentials by presynaptic somatic membrane potential. Nature 2006;441:761-765.

35 Kole MH, Letzkus JJ, Stuart GJ: Axon initial segment Kv1 channels control axonal action potential waveform and synaptic efficacy. Neuron 2007;55:633-647.

36 Zhao S, et al: Cell type-specific channel rhodopsin-2 transgenic mice for optogenetic dissection of neural circuitry function. Nat Methods 2011;8:745-752.

37 Alle H, Geiger JR: Combined analog and action potential coding in hippocampal mossy fibers. Science 2006;311:1290-1293.

38 Duan W, et al: A mathematical model of adult GnRH neurons in mouse brain and its bifurcation analysis. J Theor Biol 2011;276: $22-34$.

39 Lee K, et al: Two slow calcium-activated afterhyperpolarization currents control burst firing dynamics in gonadotropin-releasing hormone neurons. J Neurosci 2010;30:62146224. 
40 Chen X, et al: Regulation of electrical bursting in a spatiotemporal model of a GnRH neuron. Bull Math Biol 2013;75:1941-1960.

41 Roberts CB, O’Boyle MP, Suter KJ: Dendrites determine the contribution of after depolarization potentials (ADPs) to generation of repetitive action potentials in hypothalamic gonadotropin releasing-hormone $(\mathrm{GnRH})$ neurons. J Comput Neurosci 2009;26:39-53.

42 Chu Z, Moenter SM: Physiologic regulation of a tetrodotoxin-sensitive sodium influx that mediates a slow afterdepolarization potential in gonadotropin-releasing hormone neurons: possible implications for the central regulation of fertility. J Neurosci 2006;26:1196111973.

43 Maeda K, et al: Neurobiological mechanisms underlying $\mathrm{GnRH}$ pulse generation by the hypothalamus. Brain Res 2010;1364:103-115.

44 Giri M, Kaufman JM: In vitro GnRH release from the isolated medial basal hypothalamus of the male guinea pig: evidence for the existence of two pools of releasable GnRH. Brain Res 1994;648:270-280.

45 Meyer DC: In-vitro pulsatile luteinizing hormone-releasing hormone output is dependent on hypothalamic region and the stage of the estrous cycle. Biol Reprod 1987;37:12071214.

46 Purnelle G, et al: Pulsatile secretion of gonadotropin-releasing hormone by rat hypothalamic explants without cell bodies of $\mathrm{GnRH}$ neurons. Neuroendocrinology 1997;66:305312.

\section{$>$}

47 Bourguignon JP, et al: Control of pulsatile secretion of gonadotrophin releasing hormone from hypothalamic explants. Hum Reprod 1993;8(suppl 2):18-22.

48 D’Anglemont de Tassigny X, et al: Kisspeptin can stimulate gonadotropin-releasing hormone $(\mathrm{GnRH})$ release by a direct action at GnRH nerve terminals. Endocrinology 2008; 149:3926-3932.

49 Gaskins GT, Glanowska KM, Moenter SM: Activation of neurokinin 3 receptors stimulates $\mathrm{GnRH}$ release in a location-dependent but kisspeptin-independent manner in adult mice. Endocrinology 2013;154:3984-3989.

50 Uenoyama Y, et al: Ultrastructural evidence of kisspeptin-gonadotrophin-releasing hormone interaction in the median eminence of female rats: implication of axo-axonal regulation of GnRH release. J Neuroendocrinol 2011;23:863-870.

51 Smith JT, et al: Kisspeptin is essential for the full preovulatory LH surge and stimulates $\mathrm{GnRH}$ release from the isolated ovine median eminence. Endocrinology 2011;152: 1001-1012.

52 Hoffman GE, et al: Luteinizing hormone-releasing hormone neurons express c-fos antigen after steroid activation. Endocrinology 1990;126:1736-1741.
53 Lee WS, Smith MS, Hoffman GE: Progesterone enhances the surge of luteinizing hormone by increasing the activation of luteinizing hormone-releasing hormone neurons. Endocrinology 1990;127:2604-2606.

54 Moenter SM, Karsch FJ, Lehman MN: Fos expression during the estradiol-induced gonadotropin-releasing hormone $(\mathrm{GnRH})$ surge of the ewe: induction in $\mathrm{GnRH}$ and other neurons. Endocrinology 1993;133:896-903.

55 Debanne D, Bialowas A, Rama S: What are the mechanisms for analogue and digital signalling in the brain? Nat Rev Neurosci 2013;14: 63-69.

56 Semyanov A, Kullmann DM: Kainate receptor-dependent axonal depolarization and action potential initiation in interneurons. Nat Neurosci 2001;4:718-723.

57 Dellal SS, Luo R, Otis TS: GABA receptors increase excitability and conduction velocity of cerebellar parallel fiber axons. J Neurophysiol 2012;107:2958-2970.

58 Werblin FS, Dowling JE: Organization of the retina of the mudpuppy, Necturus maculosus. II. Intracellular recording. J Neurophysiol 1969;32:339-355.

59 Marder E: Neurobiology: extending influence. Nature 2006;441:702-703.

-60 Shimahara T, Peretz B: Soma potential of an interneurone controls transmitter release in a monosynaptic pathway in Aplysia. Nature 1978;273:158-160. 